\title{
Ethical guidelines for positive psychology practice
}

Aaron Jarden · Tayyab Rashid · Annalise Roache · Tim Lomas 


\section{Contents}

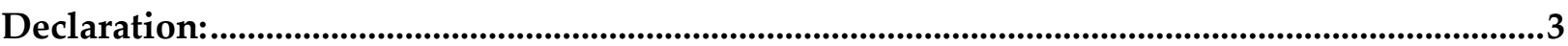

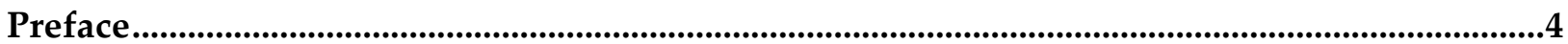

Iteration two: An overview of the new structure, content changes, and the review process................ 4

A call for support from the positive psychology community...............................................................

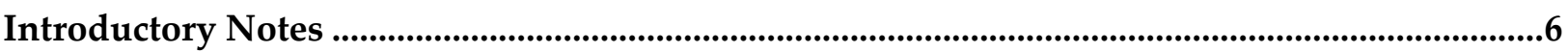

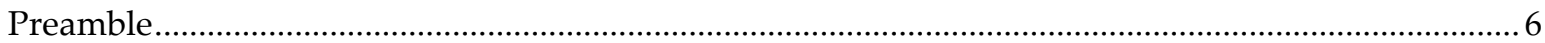

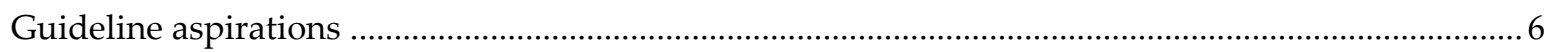

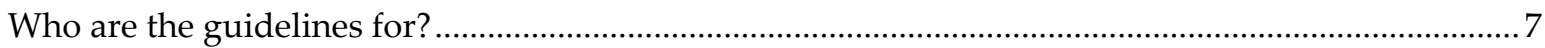

Professional bodies and the ethical guidelines for positive psychology practice …...............................

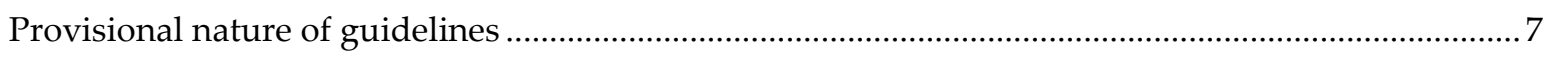

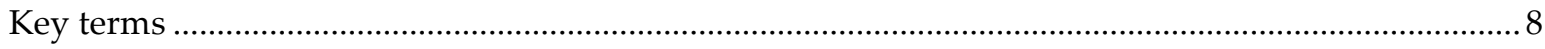

Ethical Guidelines: Values, Strengths, and Principles..............................................................10

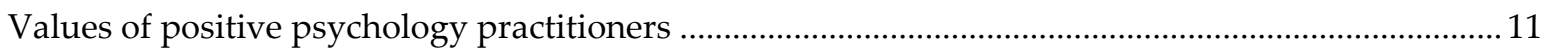

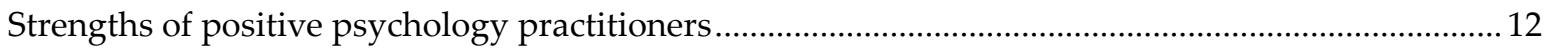

Ethical principles for positive psychology practice................................................................................ 13

Acknowledgements......................................................................................................................................15

Signatories to the Ethical Guidelines for Positive Psychology Practice ........................................17

Authors

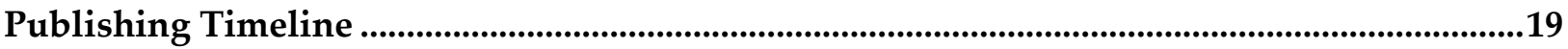

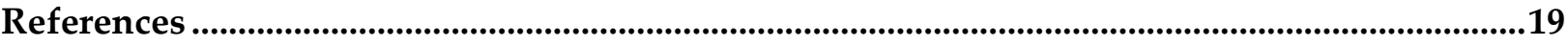

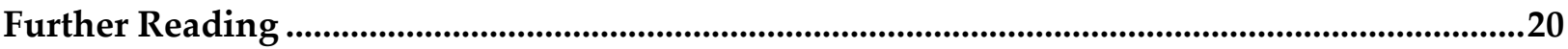

Appendix A: Applications of the Ethical Guidelines in Practice ....................................................21

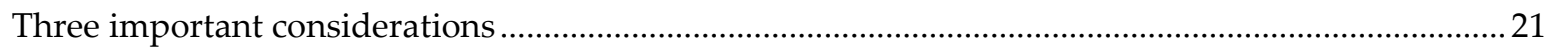

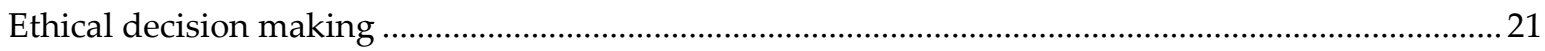

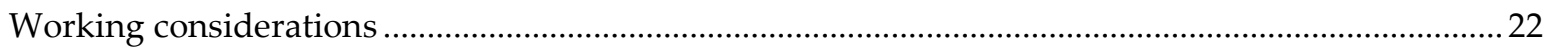

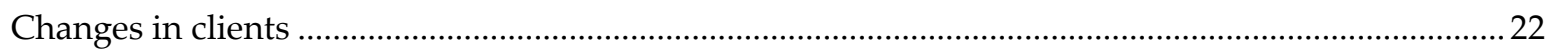

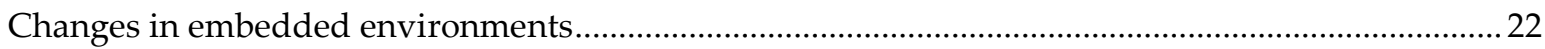

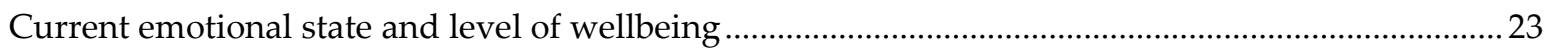

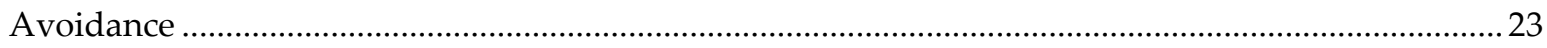

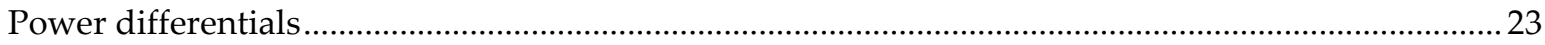

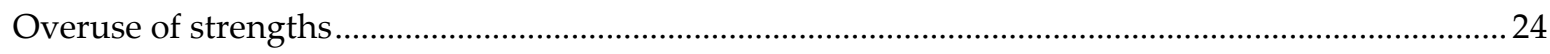

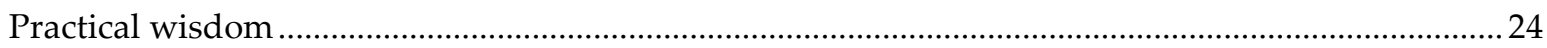

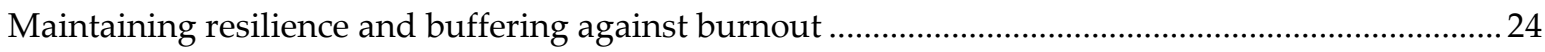

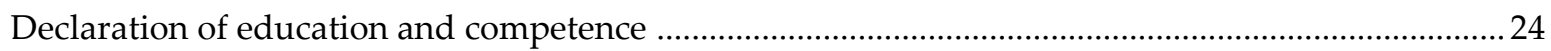

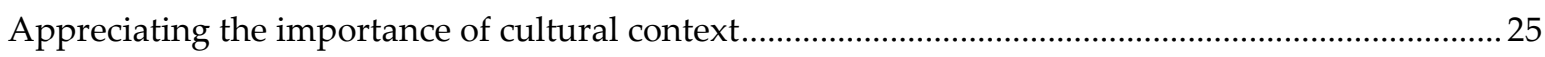

Appendix B: Resolving Ethical Dilemmas - A Step-By-Step Guide ...............................................26

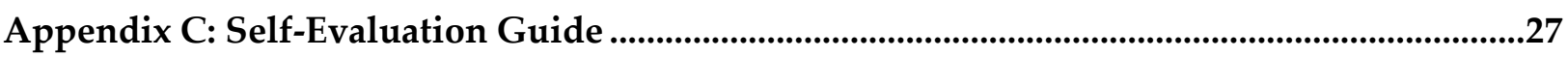

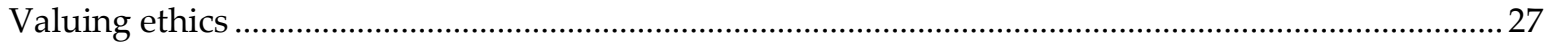




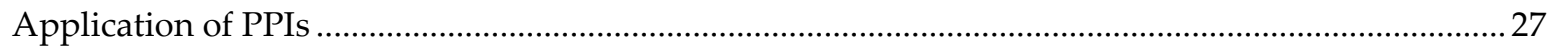

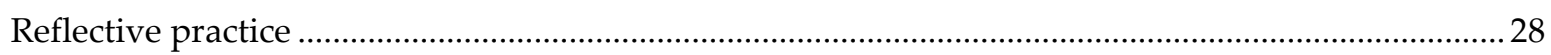

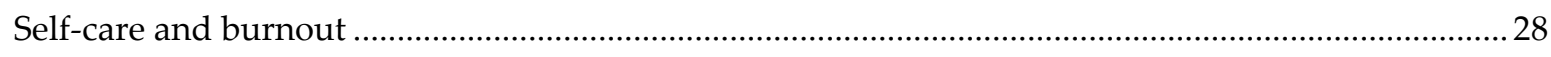

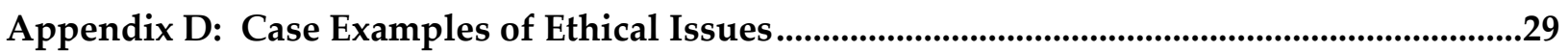

Case example 1: Cultural - applying PPIs with cultural sensitivity ..................................................29

Case example 2: Coaching - coaching clients in changing circumstances ..........................................30

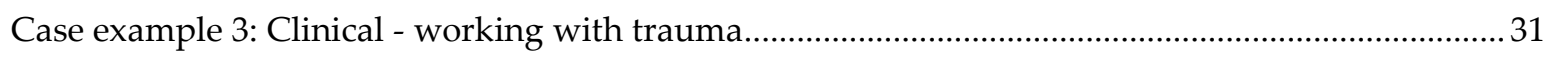

Case example 4: Organisational - pressure to misrepresent data....................................................3 31

Case example 5: Mentoring - motivating "unwilling" participants in an imbalanced relationship.. 32

Case example 6: Education - implementing wellbeing programmes where aspirations are hindered

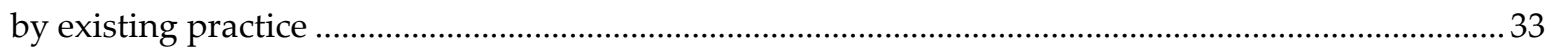

Case example 7 : Making statements about one's qualifications and about applied research ............34

Appendix E: Moving Towards a ‘Code of Ethical Positive Psychology Practice’?......................36

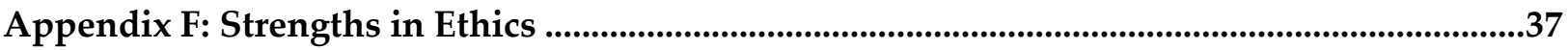

Considerations for using strengths to support ethical decision making...........................................37

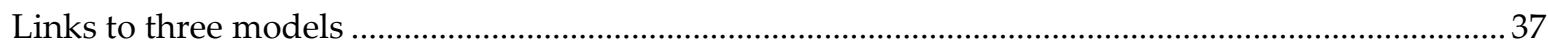

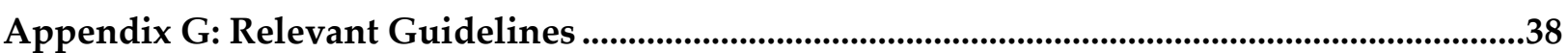

Declaration: These guidelines are the result of a collaborative and independent working group led by Aaron Jarden, Tayyab Rashid, Annalise Roache and Tim Lomas. The guidelines are independent of any organisation or association; however, numerous parties have been involved in the development and refinement of both the first and this, the second, iteration. It is the authors' intention to update the guidelines on a bi-annual cycle to further strengthen their depth and breadth of functionality, and we welcome feedback from the community to aaron.jarden@unimelb.edu.au or ethicalguidelinesppp@gmail.com. 


\section{Preface}

Iteration two: An overview of the new structure, content changes, and the review process.

The authors are dedicated to ensuring that these guidelines are relevant and accessible globally in the contexts where positive psychology is practised. As such, we have committed to a twoyearly review of the guidelines to assure there is an opportunity to refine and expand the guidelines to ensure that they meet the needs of our diverse community. The process for the review of iteration one started in July 2020. Feedback received has been both formal and informal. Informally we heard from interested parties who have reviewed the guidelines in settings like academic peer book clubs, teachers using the guidelines with their students and other interested academics, scholars and practitioners. Formally we undertook a process of invited feedback. We asked people to complete a survey, provide more in-depth feedback through open-ended questions, or directly contact us. We asked those invited individuals to pass on the invitation to anyone they felt might like to be involved, creating a snowball effect. We sought feedback from Masters in Applied Positive Psychology (MAPP) programme leaders, those who had endorsed and helped create iteration one (including some ethicists), people who showed interest, those who teach positive psychology at a university level, the translation teams, and relevant organisation representatives of practitioners using positive psychology coaching and positive psychology training providers.

The feedback received was critical, largely positive, and constructive, and resulted in several changes to the first iteration. In addition to this, we did receive a few comments that were considered beyond the scope of the current review, such as queries about "who gets to be a positive psychology practitioner?" and possible additional sections, such as 'Ethical Guidelines for Conducting Positive Psychology Research'.

Iteration two contains several fundamental changes, which are both structural and content related. Firstly, we developed and amended many aspects of the content with a particular focus on expanding and refining the language used to ensure that the guidelines, and the practitioners using them, are taking into consideration the needs of different populations (e.g., age, gender, sexuality and gender identity, neurodiversity), cultural awareness and diverse applications of positive psychology.

Secondly, we undertook major content reorganisation, moving to a more condensed 'core' document which contains the introductory notes and the Ethical Guidelines values, strengths and principles for positive psychology practice. The remainder of the content, which is more supplementary, along with new material, is now housed in a series of supporting appendices. These include sections covering; the application of ethical guidelines in practice, a step-by-step guide to resolving ethical dilemmas, an expanded self-evaluation guide, and case studies (including one new example). Two new appendices include an overview of what moving towards a 'Code of Ethical Positive Psychology Practice' could look like and a section on using strengths to support ethical decision making.

Aaron Jarden, Tayyab Rashid, Annalise Roache, Tim Lomas 
A call for support from the positive psychology community.

We invite your feedback and thoughts about the guidelines, their usefulness, and any gaps (i.e., in the application, language use, cultural inclusivity) that may benefit from being addressed.

We also welcome ideas for new content and written submissions you believe would be helpful for users of the guidelines. Feedback is open for 12 months (closing July 2022).

We also seek your assistant in ensuring these guidelines are shared with those who could most benefit from their guidance. Please contact us if you have ideas about how to extend their usefulness and reach.

Submit feedback via the survey https://www.surveymonkey.com/r/ethicalguidelinesfeedback or scanning the QR code. If you would like to contact the authors directly, please email to aaron.jarden@unimelb.edu.au or ethicalguidelinesppp@gmail.com.

A note of thanks to the translators.

Lastly, we would like to pay special thanks to the many talented individuals who have taken the time to translate these guidelines into 10 additional languages which include;

Johannes Heekerens, Aljoscha Dreisörner (German)

Natalie Zadykian, Natalia Tsvetkova (Russian)

Carina Bidese, Vivian Callegaro, Rafael Ruschel (Portuguese)

Marjan HassaniRaad, Naghmeh Taghva (Persian)

Andrea Giraldez-Hayes (Spanish)

Julie Beicker, Jannie Stricker (Danish)

Sok-ho Trinh, Hrafnhildur Krumma Jonsdottir (French)

Aisha AlAhmadi (Arabic)

Gulten Adiguzel (Turkish)

Virginia Karavakou, Christina Seryianni (Greek)

We hope to expand the translations offered to as many languages as possible to ensure these guidelines are accessible wherever positive psychology is being applied across cultures. If you are interested in translating the guidelines, please contact Aaron Jarden at aaron.jarden@unimelb.edu.au 


\section{Introductory Notes}

\section{Preamble.}

Ethical perfection may be a utopian ideal, since human beings, including positive psychology practitioners (PPPs), are fallible, vulnerable, and imperfect. Moreover, the complexities of contemporary life-including ever-expanding cyber-living, erratic climate change, global pandemics, refugee crises, evolving identities, and increasing economic polarisation exacerbate such human vulnerabilities. Psychological services, especially explicitly aiming to restore or enhance wellbeing, are not easily offered without competing priorities and ethical dilemmas. No single set of ethical guidelines, standards, codes or even statutes can fully encapsulate the range of human complexities. Nevertheless, we believe that a broad framework of values, personal strengths, and principles, nonetheless can guide us to more and better ethical decision making. This principle applies in all domains of endeavour, not least - most relevantly here - in engaging in positive psychological interventions (PPIs), which inherently aim to enhance wellbeing.

This guideline contains a) values, b) strengths, and c) principles for positive psychology practice. It is important to note that 'guidelines' usually sit within broader frameworks, of ethical practice, which will vary by context and jurisdiction. For example, guidelines may sit at the bottom of a prescriptive hierarchy such as that depicted in Figure 1 below:

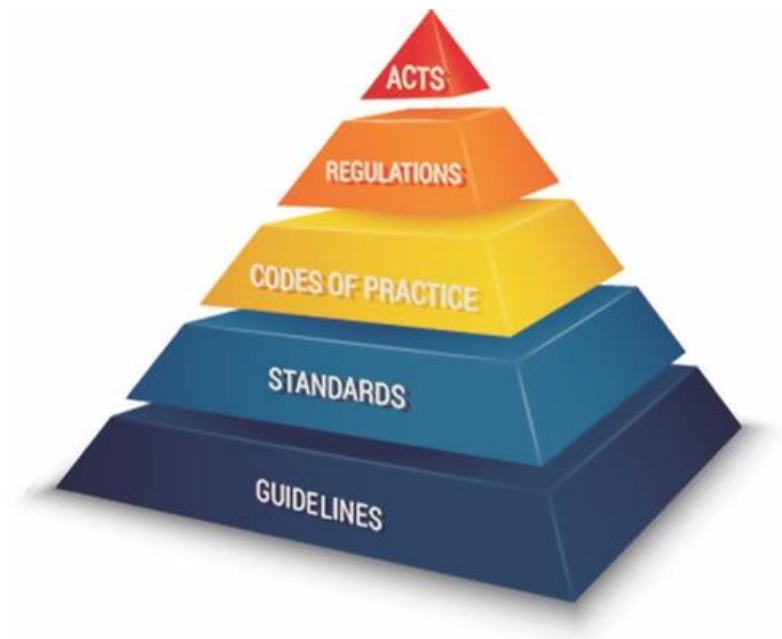

Figure 1. Guidelines and broader frameworks.

Thus, it is important to consider these guidelines along with other relevant frameworks. In addition, whilst an individual or organisation may subscribe to and endorse these guidelines for "positive psychology practice," in some countries and jurisdictions it may be illegal to call yourself a "positive psychologist" - please be aware of the restrictions and requirements of your location.

\section{Guideline aspirations.}

Why have a set of ethical guidelines? The purpose of these guidelines is to maintain and enhance ethical practice upholding a commitment to scientifically informed and evidencebased practice in the field of positive psychology (PP). At the highest professional level they aim to inspire fidelity to the science of wellbeing, and practitioners will ideally consider the guidelines in their self-presentation and marketing of the potential benefits. With the intent to 
protect the public against potential harmful impact-intentional or inadvertent. The guidelines attempt to promote to PPPs and others a clearer understanding of what constitutes the ethically informed practice of PP. With the assistance of such guidelines, PPPs are expected to uphold commitment to aspects such as: ${ }^{1}$

a) closely studying relevant research, and building their practice from a scientific base;

b) continually updating their knowledge through ongoing learning and professional development;

c) carefully and accurately communicating the limits of knowledge related to PPIs, as well as potential benefits and pitfalls of specific PPIs and wellbeing programmes; and

d) monitoring the wellbeing of their clients during service provision, and especially any negative impacts on wellbeing.

Who are the guidelines for?

The purpose of these guidelines is to facilitate optimal ethical practice of PPPs, doing so by encouraging PPPs to act beneficently (doing good to others) and non-maleficently (avoiding potential harm to others). As such, these guidelines are for professionals (e.g., psychologists) and others (e.g., students, or those with no affiliations) who are delivering PPIs or using knowledge from the field of PP in their practice. These are not guidelines for how to use or deliver particular PPIs.

Professional bodies and the ethical guidelines for positive psychology practice.

Where a practitioner is a member of a relevant professional body (e.g., American Psychological Association, Australian Psychological Society, International Coaching Federation), then first and foremost, their association's standards, code or guidelines will inform their practice. We further expect that when practitioners are members of a licensing or credentialing body (e.g., College of Psychologists) - with adequate education, training and experience in practising psychology or related fields - such practitioners will therefore be familiar with the rules, standards, regulations, statues, and procedures of practising psychology within their jurisdiction.

The ethical guidelines for positive psychology practice introduced here are designed to augment, not replace, such existing jurisdictional/professional codes and guides. As such, these guidelines also provide a baseline for practitioners who are not associated with or affiliated to a professional body. Furthermore, countries or regions of the world which may not have any coherent set of guidelines of practice of psychology, these Guidelines can help practitioners to inform or organise their practice on ethical principles.

\section{Provisional nature of guidelines.}

We note that the existing published guidelines (version 1.0, 19 $9^{\text {th }}$ July 2019), and this new iteration (version 2.0, 16 July, 2021) are provisional and subject to ongoing revision every two years through an iterative consultative process with key stakeholders. Such regularity is to ensure that the guidelines are responsive to contextual changes and reflect ever evolving local

\footnotetext{
${ }^{1}$ We have chosen four examples here to demonstrate such commitments, however, note that this list could be lengthy.
} 
and global themes. In that respect, we welcome feedback on the guidelines outlined here at any time.

It is also important to note that primarily English speaking, mostly Western countries, have influenced the development of these guidelines. As such, although we have sought to make the guidelines universally relevant, they may potentially be limited to such contexts. However, since these guidelines are a living growing document identified by version number, it is hoped that future versions will incorporate the influence of codes and guidelines from non-English speaking and non-Western countries.

Final note - This is an iterative process.

We remind the reader of these guidelines that they have been designed to be living and evolving, and as such, are both imperfect and intended to be updated every two years. Feedback from all stakeholders will be reviewed by the custodians (authors) and will be considered for each future iteration.

\section{Key terms.}

\section{Client}

Practitioners work with clients. "Client" is a generic term used to refer to the recipient of any services such as (however, not limited to) an individual, couple, family, group, organisation, sponsor, or other specifiable social unit.

\section{Codes vs guidelines}

A code usually has legal or other enforceable ramifications, whereas guidelines are limited to suggesting best practice which is not usually "regulated" or "policed." Guidelines, by their nature, imply that circumstances are varied and complex, and that following the guidelines often requires practitioners to apply practical wisdom (a combination of experience and common sense), as well as to abide by relevant other local and professional codes. See Appendix E for more on what developing a code may look like.

$\underline{\mathrm{PP}}$

PP: Positive psychology. There are many ways of conceptualising and defining PP, but most operationalisations usually position it as the scientific study and promotion of overarching constructs such as "happiness," "wellbeing," "flourishing," and "thriving." For instance, Lomas, Hefferon and Ivtzan (2015, p. 1347) define PP as "the science and practice of improving wellbeing." Relatedly, there is the notion of "applied positive psychology," which can simply be read as the application of PP in real-world settings, including through PPIs.

$\underline{\text { PPI }}$

PPI: Positive psychological intervention. There is within the literature a demarcation made between a "positive psychology intervention" and a "positive psychological intervention" - for an outline and explanation, see Hone, Jarden, and Schofield (2015). Here we incorporate the wider notion of positive psychological intervention that covers all activities across many disciplines (i.e., from other sub-fields of psychology in addition to PP) aimed towards increasing psychological forms of wellbeing. One popular definition of a PPI is: “A positive psychological intervention promotes positive emotions, behaviours, and/or thoughts, thereby increasing the 
wellbeing of an individual or group" (Schueller, Kashdan, \& Parks, 2014, p. 92). Another popular definition of a PPI is "treatment methods or intentional activities that aim to cultivate positive feelings, behaviours, or cognitions" (Sin \& Lyubomirsky, 2009, p. 468).

$\underline{\text { PPP }}$

PPP: Positive psychology practitioner. A practitioner who has completed requisite training to practise (e.g., psychotherapy, counselling, coaching), and undertaken PP specific training with a reputable training provider, thereby allowing an understanding of the scientific research process and its ethical application in the delivery of applied positive psychology (e.g., PPIs). ${ }^{2}$

\section{Strengths}

Generally, ethical guidelines and codes espouse specific desired personal characteristics, arbitrarily selected by thought leaders of the specific field. For these guidelines, we reviewed salient frameworks of strengths (Appendix F). Rather than synthesizing these diverse frameworks into one unified model of strengths, which is beyond the scope of these guidelines, we elected to work from one framework based on available empirical evidence (McGrath, 2016; McGrath, 2015; McGrath, 2014; Peterson \& Seligman, 2004). The VIA Classification of Character Strengths and Virtues (Peterson \& Seligman, 2004) presents the strongest empirical evidence and application across various domains such as educational, clinical, workplace, health and leisure. According to the VIA, character strengths are defined as universal traits that are valued in their own right and do not necessarily lead to instrumental outcomes. Rather, as behavioural traits, they facilitate and guide a person to behave in responsible and ethical ways for their own and others' welfare. Character strengths, broadly speaking, reflect who a person is (i.e., their character), and values reflect what a person is committed to (i.e., their principles and actions).

\section{Practitioner}

The term "practitioner" is used generically to refer to anyone with responsibility for the provision of PP related services. "Practitioner" includes anyone undertaking role(s) such as (though not limited to) counsellor, psychotherapist, mental health professional, coach, trainer, mentor, or educator. ${ }^{3}$

\section{Principles}

Principles direct attention to important ethical responsibilities. Particular actions follow from specific principles.

\section{$\underline{\text { Values }}$}

Values are beliefs held by individuals and shared by groups about desirable ends (i.e., broader ideologies about the world and how it should be). Values transcend specific situations, guide

\footnotetext{
2 Who can call themselves a PPP? There is, as yet, no clear demarcation as to the amount and level of training or qualification needed. For example, could one consider oneself a PPP based on a 2-day course, 6-month accredited certificate, or a 2-year Masters in Applied Positive Psychology Programme? These are questions that still need resolving within the field, and are beyond the scope of these guidelines per se. In addition, supervised practice (e.g. peer and one-to-one supervision) and online consultation is ideal. Similarly, further guidance regarding appropriate training and supervision is needed from the professional bodies in this field (and indeed, such issues are already being considered and addressed by relevant parties).

${ }^{3}$ The ethos and principles contained in these guidelines could equally apply to wellbeing researchers, where researchers could be considered practitioners in one sense, working with subjects who could be considered similar to clients.
} 
how we select actions and evaluate others and ourselves, and are ordered by their relative importance (Schwartz, 2006). Values can represent an important way of expressing a general ethical commitment that becomes more precisely defined and action-orientated when expressed as a principle. In other words, values inform principles. In that communal sense, the values outlined in these guidelines are those the authors have identified as being broadly shared within the PP community. We also acknowledge that different values may vary in importance in different contexts.

\section{Ethical Guidelines: Values, Strengths, and Principles}

The ethical guidelines for positive psychology practice incorporate the following values, strengths and principles. Both values and strengths support the enactment of ethical principles, as displayed in Figure 1 and expanded on in further detail in Table 1.

\section{Table 1}

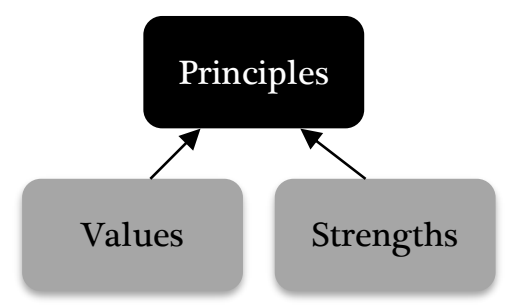

Values, Strengths and Principles of Positive Psychology Practice.

\begin{tabular}{|c|c|c|}
\hline Values $^{4}$ & Strengths 5 & Principles \\
\hline - Protecting the safety of clients and others. & - Honesty. & $\begin{array}{l}\text { - Beneficence/non- } \\
\text { maleficence. }\end{array}$ \\
\hline - Alleviating personal distress and suffering. & - Fairness. & - Responsible caring. \\
\hline $\begin{array}{l}\text { - Ensuring the integrity of practitioner-client } \\
\text { relationships. }\end{array}$ & - Social intelligence. & $\begin{array}{l}\text { - } \begin{array}{l}\text { Respect for people's } \\
\text { rights and dignity. }\end{array}\end{array}$ \\
\hline $\begin{array}{l}\text { - Appreciating the diversity of human } \\
\text { experience and culture. }\end{array}$ & - Teamwork. & - Trustworthiness. \\
\hline $\begin{array}{l}\text { - Fostering a sense of self that is meaningful } \\
\text { to the person(s) concerned. }\end{array}$ & - Kindness. & - Justice. \\
\hline $\begin{array}{l}\text { - Enhancing the quality of professional } \\
\text { knowledge and its application. }\end{array}$ & - $\quad$ Prudence. & - Autonomy. \\
\hline $\begin{array}{l}\text { - Enhancing the quality of relationships } \\
\text { between people. }\end{array}$ & - Perspective. & \\
\hline - Increasing personal effectiveness. & ○ Judgement. & \\
\hline \multirow[t]{3}{*}{$\begin{array}{l}\text { - Striving for the fair and adequate } \\
\text { provision of counselling, psychotherapy, } \\
\text { coaching, and wellbeing services. }\end{array}$} & $\circ$ Self-regulation. & \\
\hline & ○ Perseverance. & \\
\hline & ○ Bravery. & \\
\hline
\end{tabular}

\footnotetext{
${ }^{4}$ Our values have been selected based on other similar guidelines, and, as such, many of the values can fit in different categories. For example, justice could be perceived as a value, or as a principle. Trustworthiness could be perceived as a strength (honesty), a value, or as a principle. Many of these constructs are debatable in this way. Values have also been ranked by perceived importance, according to the guideline authors.

${ }^{5}$ Strengths are sourced from 10 similar codes of ethics (e.g., British Association for Counselling and Psychotherapy's ethical framework for good practice in counselling and psychotherapy). The Strengths of Judgement, Self-regulation, Perseverance and Bravery (hollow bullet points) have been chosen by the authors as deemed important in upholding the six principles.
} 
In addition:

- Almost all codes stipulate a range of personal traits and qualities, whereas these guidelines focus on strengths and observable behaviours.

- The lists of values, strengths, and principles are not in any way exhaustive, but, rather, an initial attempt at surveying what is currently perceived most pertinent to practice. As the guidelines adapt iteratively over time, and debate is had amongst the PP community, it is anticipated that these elements will evolve. We note that the content of Table 1 (Values, Strengths, Principles), remain the same in version two as they were in version one.

- The lists of values, strengths and principles is also not prescriptive.

- One particular model of strengths is used here: the VIA Classification of Character Strengths and Virtues framework. This selection is based on the VIA being deemed to have the largest empirical research base in the literature among comparable frameworks. However, if you have a personal preference for, and familiarity with, a different strengths framework (e.g., Gallup CliftonStrengths), then we encourage you to use that instead. In that case, aim to map the relevant strengths from your preferred framework onto the VIA strengths listed (e.g., for the first strength of 'honesty,' substitute in the nearest equivalent strength from your preferred framework). The general principle here is to use strengths as such in acting ethically, not necessarily to use the strengths specified by any particular framework.

Values of positive psychology practitioners.

The fundamental values of PPPs include a commitment to:

- Protecting the safety of clients and others. This includes maintaining an ever-vigilant awareness of how a client is responding to one's services, and, moreover, recognising the limits of one's capacity and expertise (and, as such, recognising when a client may need professional help from other sectors, or if the public are in danger).

- Alleviating personal distress and suffering (when within one's boundaries of expertise). This means deploying PPIs in contextually relevant ways to decrease the distress of individuals who seek services in various professional contexts (e.g., therapy, coaching, health).

- Ensuring the integrity of practitioner-client relationships. This includes respecting personal boundaries, and maintaining a commitment to professional practices such as consent, confidentiality, and objectivity.

- Appreciating the diversity of human experience and cultures. This means not applying a "one-size-fits-all" framework to one's clients, but, rather, respecting and indeed celebrating the variety of people's unique developmental paths, and contextual and cultural backgrounds.

- Fostering a sense of self that is meaningful to the person(s) concerned. This means that the individual develops and pursues goals that are not only fulfilling for the self, but also contribute to the greater good.

- Enhancing the quality of professional knowledge and its application. This means doing one's best to stay abreast of developments in the research literature and maintaining an up-to-date knowledge of advances in the field. 
- Enhancing the quality of relationships between people. This means recognising that people are inevitably situated within social relationships and networks, and striving to help the person enhance these bonds as far as possible.

- Increasing personal effectiveness. This means striving to help the client operate more effectively in the world and in the unique situations they find themselves.

- Striving for the fair and adequate provision of PP services. This might include seeking to provide services for hard-to-reach populations, or, where possible, implementing a pricing structure whereby people with less means are still able to benefit from services.

\section{Strengths of positive psychology practitioners.}

The practitioner's personal strengths are essential because they have an impact on the practitioner/client relationship. Many of the strengths considered vital in the provision of services are invariably deemed good personal qualities in life generally. However, it is yet inappropriate to prescribe that all practitioners possess these strengths since it is fundamental that these are deeply rooted in the person concerned and develop out of personal commitment rather than the requirements of an external authority. A thematic analysis of principles and values espoused by major guidelines (see Relevant Guidelines section) showed that the following character strengths are presumed to facilitate sound ethical practice and decision making:

- Honesty: Speaking the truth; presenting oneself in a genuine way and acting in a sincere way; being without pretence; taking responsibility for one's feelings and actions.

- Fairness: Treating all people the same according to notions of fairness and justice; not letting personal feelings bias decisions about others; giving everyone a fair chance.

- Social Intelligence: Being aware of the motives and feelings of other people; knowing what to do to fit into different social situations; knowing what to do to put others at ease.

- Teamwork: Working well as a member of a group or team; being loyal to the group; doing one's share.

- Kindness: Doing favours and good deeds for others; helping them; taking care of them.

- Prudence: Being careful about one's choices; not taking undue risks; not saying or doing things that might later be regretted.

- Perspective: Being able to provide wise counsel to others; having ways of looking at the world that make sense to oneself and to other people.

- Judgement: Thinking things through and examining them from all sides; not jumping to conclusions; relying only on solid evidence to make decisions; having the ability to change one's mind.

- Self-regulation: Regulating what one feels and does; being disciplined; controlling one's appetites and emotions.

- Perseverance: Working hard to finish what one starts, no matter the project: "getting it out the door" in timely fashion; not getting distracted when working; taking satisfaction in completing tasks.

- Bravery: Being a courageous person who does not shrink from threat, challenge, difficulty, or pain; speaking up for what is right even if there is opposition; acting on one's convictions. 
Ethical principles for positive psychology practice.

Each of the six ethical principles described below is accompanied by examples of good practice that have been developed in response to that principle. Ethical decisions that are strongly supported by one or more of these principles, without any contradiction from others, may be regarded as having a strong moral rationale. However, practitioners will likely encounter circumstances in which it is impossible to reconcile all the applicable principles, and choosing between principles may be required. A decision or course of action does not necessarily become unethical merely because it is contentious or other practitioners would have reached different conclusions in similar circumstances. A practitioner's obligation is to consider all the relevant circumstances with as much care as is reasonably possible and to be appropriately accountable for decisions made.

\section{Beneficence/non-maleficence}

Beneficence involves a commitment to promoting the client's and practitioner's wellbeing. The principle of beneficence means acting in the best interests of the client, based on professional assessment. It directs attention to working strictly within one's limits of competence and providing services on the basis of adequate training or experience. Ensuring that the client's best interests are achieved requires systematic monitoring of practice and outcomes (e.g., wellbeing) by the best available means. It is considered important that scientific research and systematic reflection inform practice. Depending on role and context, there is usually an obligation to take steps to enhance the quality of the services provided, and to commit to updating practice by continuing professional development. An obligation to act in the best interests of a client may become paramount when working with clients whose capacity for autonomy is diminished because of age, immaturity, lack of understanding, distress, or other significant personal constraints.

Non-maleficence, on the other hand, involves a commitment to avoiding harm to the client. Practitioners who adopt this principle:

- avoid sexual, financial, emotional or any other form of client exploitation;

- avoid incompetence (i.e., appropriate application of PPIs, e.g., knowing what works for whom) and malpractice;

- do not provide services when unfit to do so due to illness, personal circumstances or intoxication.

The practitioner has an ethical responsibility to strive to mitigate any harm caused to a client even when the harm is unavoidable or unintended. Practitioners have personal and professional responsibilities to challenge, where appropriate, the incompetence or malpractice of others. They also have a responsibility to contribute to any investigation, and/or adjudication by an appropriately recognised body/authority, concerning professional practice which falls below that of a reasonably competent practitioner and/or risks bringing discredit upon their profession or PP.

\section{Responsible caring}

The practice of PP promotes wellbeing. In pursuing this goal, PPPs who adopt this principle demonstrate an active concern for the welfare of those with whom they work, and acknowledge the social and institutional power that structures their role as PPPs. They have a 
primary responsibility to protect the welfare of those with whom they work. They recognise that individuals, families, groups, or communities may be in a vulnerable position. They also recognise their boundaries of expertise (including the absence of knowledge) and refer to other specialists when needed, and they accept responsibility and correct any harm that occurs as a result of their service provision. PPPs are also expected to take an active and ongoing interest in empirical and theoretical developments in their field, ensuring that they maintain up-to-date knowledge of the evidence base regarding the PPIs they are implementing.

\section{$\underline{\text { Respect for people's rights and dignity }}$}

In accordance with the United Nations Universal Declaration of Human Rights, respect for the dignity of persons and their rights requires that: each person and all peoples are positively valued in their own right, their rights are acknowledged and respected, and they are shown respect and granted dignity as part of their common humanity. Practitioners who adopt this principle show respect which requires sensitivity to cultural and social diversity. This includes a recognition of differences among persons associated with their culture, nationality, ethnicity, colour, race, religion, gender, marital status, sexual orientation, physical or mental abilities, age, socio-economic status, and/or any other personal characteristic, condition, or status. Such differences may be an integral part of the person, and should be respected as such.

\section{Trustworthiness}

Being trustworthy (fidelity) involves honouring the trust placed in the practitioner.

Trustworthiness is regarded as fundamental to understanding and resolving ethical issues. Practitioners who adopt this principle:

- act in accordance with the trust placed in them;

- strive to ensure that clients' expectations have reasonable prospects of being met;

- honour their agreements and promises;

- regard confidentiality as an obligation arising from the client's trust;

- restrict any disclosure of confidential information about clients to furthering the purposes for which it was originally disclosed - with the exception of mandated disclosure (e.g., a law in one's jurisdiction requires such disclosure).

Justice

Justice involves the fair and impartial treatment of all clients and the provision of adequate services. The principle of justice requires that practitioners who adopt this principle are just and fair to all clients, including hard-to-reach populations, and respect their human rights and dignity. It directs attention to conscientiously considering any legal requirements and obligations, and remaining alert to potential conflicts between legal and ethical obligations. Justice in the distribution of services requires the ability to determine impartially the provision of services for clients and the allocation of services between clients. A commitment to fairness requires the ability to appreciate differences between people and to be committed to equality of opportunity, and avoid discrimination against people or groups based on their personal or social characteristics. In other words, justice strives to ensure that unfair discrimination based on certain characteristics (e.g., gender, age, sexual orientation) does not happen. Practitioners have a duty to strive to ensure a fair provision of services that are accessible and appropriate to the needs of potential clients. 


\section{Autonomy}

Autonomy involves respecting the client's right to be self-governing. This principle emphasises the importance of developing a client's ability to be self-directing within the provision of services and all aspects of life. The principle of autonomy opposes the manipulation of clients against their will, even for beneficial social ends. Practitioners who respect their clients' autonomy:

- promote autonomy;

- safeguard and ensure independent decision making;

- recognise when the client is unable to exercise autonomy;

- ensure accuracy in any advertising or information given in advance of services offered;

- seek freely given and adequately informed consent throughout the relationship;

- emphasise the value of voluntary participation in the services being offered;

- engage in explicit contracting in advance of any commitment by the client and revisit/review the terms of that contract throughout the relationship;

- $\quad$ protect privacy;

- protect confidentiality;

- customarily make any disclosures of confidential information conditional on the consent of the person concerned;

- inform the client in advance of foreseeable conflicts of interest, or as soon as possible after such conflicts become apparent.

\section{Acknowledgements}

The following people, listed alphabetically, contributed substantially and provided input and feedback into the drafting of these guidelines:

- Associate Professor Aaron Jarden, University of Melbourne, Australia.

- Mr Andrew Alexandra, University of Melbourne, Australia.

- Annalise Roache, Auckland University of Technology, New Zealand.

- Dr Dan Weijers, University of Waikato, Hamilton, New Zealand.

- Dr Denise Quinlan, New Zealand Institute of Wellbeing and Resilience, New Zealand.

- Professor Dianne Vella-Brodrick, University of Melbourne, Australia.

- Giselle Timmerman, Positive Work, United States of America.

- Hein Zegers, European Network of Positive Psychology.

- Dr Helena Agueda Marujo Instituto Superior de Ciências Sociais e Politicas (School of Social and Political Sciences), University of Lisbon, Portugal.

- Professor James Pawelski, University of Pennsylvania, United States of America.

- Dr Jo Mitchell, The Mind Room, Melbourne, Australia.

- Dr Joel Milam, University of Southern California, USA.

- Dr Judy Moskowitz, Northwestern University Feinberg School of Medicine, United States of America.

- Associate Professor Karena Burke, Central Queensland University, Australia.

- Lisa Sansom, LVS Consulting, Canada.

- Margarita Tarragona, Positivamente, Mexico.

- Matthew Iasiello, Wellbeing and Resilience Center, SAHMRI, Australia.

- Dr Mohsen Joshanloo, Keimyung University, South Korea.

- Reb Rebele, University of Melbourne, Australia.

- Dr Robert Biswas-Diener, Positive Acorn. 
- Associate Professor Robert Isler, Waikato University.

- Dr Rona Hart, former Director MAPP University of East London, England.

- Dr Ryan Niemiec, VIA Institute on Character.

- Scott Asalone, ASGMC Inc, USA.

- Simon Murray, Positive Education Schools Association, Australia.

- Professor Stewart Donaldson, Claremount Graduate University, United States of America.

- Sue Langley, The Langley Group, Australia.

- Dr Suzy Green, The Positivity Institute, Australia.

- Dr Tayyab Rashid, University of Toronto Scarborough, Canada.

- Tia Moin, MAPPCP, Positively Me Coaching.

- Dr Tim Lomas, University of East London, England. 


\section{Signatories to the Ethical Guidelines for Positive Psychology Practice}

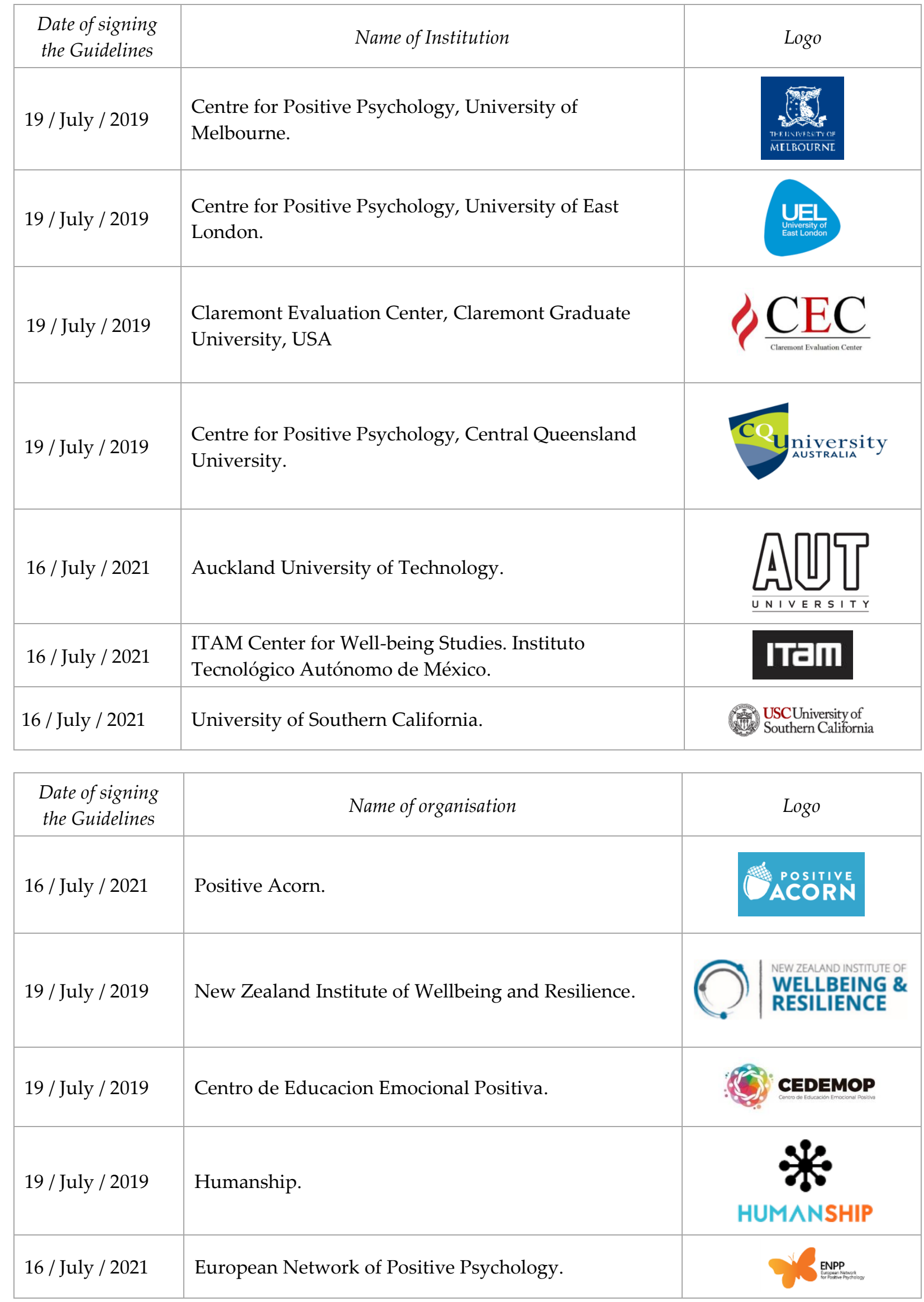




\begin{tabular}{|c|c|c|}
\hline 16 / July / 2021 & International Journal of Wellbeing. & \\
\hline 16 / July / 2021 & LVS Consulting. & $\underbrace{V}_{\text {LVSCONSULTING }}$ \\
\hline 16 / July / 2021 & The Mind Room. & \\
\hline 16 / July / 2021 & The Inclusion Coach. & $\begin{array}{l}\text { Jinclusion } \\
\text { Coach }\end{array}$ \\
\hline 19 / July / 2019 & The Langley Group. & Co Langley Group \\
\hline 16 / July / 2021 & The Positivity Institute. & \\
\hline 19 / July / 2019 & The Wellbeing and Resilience Centre. & $\begin{array}{l}\text { WELLBEING } \\
\text { ANDRESILIENCE } \\
\text { CENTRE }\end{array}$ \\
\hline 19 / July / 2019 & VIA Institute on Character. & $\begin{array}{l}\text { VIAINSTITUTEN } \\
\text { CHARACTER }\end{array}$ \\
\hline 19 / July / 2019 & Japanese Positive Health Psychology Society. & - \\
\hline 19 / July / 2019 & New Zealand Association of Positive Psychology. & \\
\hline 16 / July / 2021 & World Happiness Foundation. & $\begin{array}{l}\text { WORLD } \\
\text { HAPPNESS } \\
\text { FOUNDABIIN } \\
\text { béREATON }\end{array}$ \\
\hline \multicolumn{3}{|c|}{$\begin{array}{l}\text { * Please note that the above institutions and organisations are examples, and this is not an extensive or } \\
\text { exhaustive list. }\end{array}$} \\
\hline
\end{tabular}




\section{Authors}

\section{Aaron Jarden}

University of Melbourne, Australia

aaron.jarden@unimelb.edu.au

Tayyab Rashid

University of Toronto Scarborough, Canada

Annalise Roache

Auckland University of Technology, New Zealand

Tim Lomas

Wellbeing for Planet Earth, Japan

\section{Publishing Timeline}

Published July, 152021

\section{References}

Barlow, D. H., Allen, L. B., \& Choate, M. L. (2004). Toward a unified treatment for emotional disorders. Behavior Therapy, 35(2), 205-230.

Clifton, D. O., \& Anderson, E. (2002). Strengths Quest: Discover and develop your strengths in academics, career, and beyond. Washington, DC: The Gallup Organization.

Hone, L., Jarden, A., \& Schofield, G. (2015). An evaluation of positive psychology intervention effectiveness trials using the re-aim framework: A practice-friendly review. Journal of Positive Psychology, 10(4), 303322.

Koocher, G. P. \& Keith-Spiegel, P. (1998). Ethics in psychology: Professional standards and cases. New York, NY: Oxford University Press.

Linley, P.A. (2008). Average to A+: Realising strengths in yourself and others. Leicester: CAPP Press.

Lomas, T., Hefferon, K., \& Ivtzan, I. (2015). The LIFE model: A meta-theoretical conceptual map for applied positive psychology. Journal of Happiness Studies, 16(5), 1347-1364. https://doi.org/10.1007/s10902-0149563-y

McGrath, R. E. (2016). Measurement Invariance in Translations of the VIA Inventory of Strengths. European Journal of Psychological Assessment, 32(3), 187-194. https://doi.org/10.1027/1015-5759/a000248

McGrath, R. (2015). Character strengths in 75 nations: An update. The Journal of Positive Psychology, 10(1), 41-52. https://doi.org/10.1080/17439760.2014.888580

McGrath, R. E. (2014). Character strengths in 75 nations: An update. Journal of Positive Psychology, 10(1), 111. https://doi.org/10.1080/17439760.2014.888580

Niemiec, R. M. (2018). Character strengths interventions: A field-guide for practitioners. Boston: Hogrefe.

Peterson, C., \& Seligman, M. E. P. (2004). Character strengths and virtues: A handbook and classification. New York, NY: Oxford University Press.

Rashid, T. \& Seligman, M. E. P. (2018). Positive psychotherapy: Clinician manual. Oxford, England: Oxford University Press.

Schueller, S. M., Kashdan, T. B., \& Parks, A. C. (2014). Synthesizing positive psychological interventions: Suggestions for conducting and interpreting meta-analyses. International Journal of Wellbeing, 4(1), 9198. https://doi.org/10.5502/ijw.v4i1.5

Schwartz, S. H. (2006). Basic human values: Theory, measurement, and applications. Jerusalem, Israel: The Hebrew University of Jerusalem. 
Sin, N. L. \& Lyubomirsky, S. (2009). Enhancing well-being and alleviating depressive symptoms with positive psychology interventions: a practice friendly meta-analysis. J. Clin. Psychol. 65, 468.

van Agteren, J., Iasiello, M., Lo, L., Bartholomaeus, J., Kopsaftis, Z., Carey, M., \& Kyrios, M. (2021). A systematic review and meta-analysis of psychological interventions to improve mental wellbeing. Nature Human Behaviour, 1-22.

\section{Further Reading}

Han, H. (2014). Virtue ethics, positive psychology, and a new model of science and engineering ethics education. Science and Engineering Ethics, 21(2), 441-460.

Lomas, T., \& Ivtzan, I. (2016). Professionalising positive psychology: Developing guidelines for training and regulation. International Journal of Wellbeing, 6(3), 96-112.

Koocher, G. P., \& Keith-Spiegel, P. (2008). Ethics in psychology and the mental health professions: Standards and cases (3rd e d.). New York: Oxford University Press.

Sinclair, C. (2017). Ethics in psychology: Recalling the past, acknowledging the present, and looking to the future. Canadian Psychology, 58(1), 20-29.

Vella-Brodrick, D. (2014). Dovetailing ethical practice and positive psychology to promote integrity, industriousness, innovation, and impact. In A. C. Parks \& S. M. Schueller (Eds.), The Wiley Blackwell handbook of positive psychological interventions (pp. 416-432). Chichester, England: Wiley Blackwell. 


\section{Appendix A: Applications of the Ethical Guidelines in Practice}

This section contains several guides and examples for the contextual application of these ethical guidelines. The content is designed to deepen thinking while also providing useful tools and a self-evaluation guide for resolving and supporting ethical decision making in practice.

\section{Three important considerations.}

Before applying any specific PPIs, one should assess and appraise the following three important factors, which are the cornerstone of best practice (Barlow, Allen \& Choate, 2004):

1. Is the PPI an appropriate match for the situation? This can be ascertained by reviewing the relevant literature and published evidence from reliable sources. For example, before a specific PPI is applied for gratitude, check to see if there is existing published evidence that shows that this specific PPI is a promising technique for the particular client.

2. Does the PPI match with the client's presenting needs? For example, have aspects relevant to the person and their circumstances, such as, culture, religion, identity, motivation, been considered?

3. How will the effectiveness of a PPI be evaluated? In other words, what specific outcome can be identified and validly assessed to determine the effectiveness of the PPI?

\section{Ethical decision making.}

Ethical decision making can be assisted by: having adequate knowledge of these ethical guidelines and the guidelines of an associated profession; being aware of the virtuous ethical behaviour of others; and the use of personal strengths in decision making.

Knowledge: We expect that the primary users of these guidelines - namely, PPPs with adequate education, training, and experience in practicing psychology or related fields are familiar with and can describe these ethical guidelines and any ethical guidelines that pertain to their profession and professional standing. Most jurisdictions emphasise that practitioners need to be familiar with relevant rules, standards, regulations, statues, and procedures.

Behaviour: Ethical behaviour, especially while delivering PPIs, cannot simply rely on individual knowledge, but is guided and informed by collective behaviours and practices. Can you identify in your professional circles those individuals who model ethical practices? Although your colleagues may not explicitly highlight such practices (e.g., due to their modesty), you are encouraged to explicitly ask your peers or wider professional community (regional or international organisation) how they adhere to the ethical standards of your field. Collecting a case repository of good practices will be useful for reflexivity with respect to one's own behaviour. With sufficient accumulation of cases, illustrations of excellence will likely appear. Ideally, practitioners will create an accessible hub where other practitioners have an opportunity to enrich this repository.

Using strengths towards ethical decision making: Strengths can be helpful in several ways in ethical decision making. For example, identifying strengths exemplars can help with one's ethical decision making. Find a colleague who presents as a role model as an exemplar of ethical behaviour. Reflect how they handled a specific ethical dilemma or situation, or, more generally, which specific strengths they embody in day-to-day 
interactions with colleagues and clients that is worth modelling and may help with one's own decision making.

\section{Working considerations.}

When working with clients, there are numerous considerations to be mindful of that can have immediate and important ethical implications. In particular, it is pertinent to consider: changes in clients; changes in the environments in which PPPs and clients find themselves; the current emotional state and level of wellbeing of clients and PPPs; clients' tendency towards avoidance; relationship power differentials; overuse of strengths; practical wisdom; maintaining resilience and buffering against burnout; a declaration of education and competence; and the importance of varying cultural contexts. Consideration of these aspects can help mitigate, anticipate, or prevent some inadvertent and unpleasant consequences.

\section{Changes in clients.}

While delivering any psychological services, it is important to monitor the wellbeing of clients throughout the course of treatment or intervention. Some clients may deteriorate, despite the demonstrated effectiveness of the PPI, for any number of reasons, making clients vulnerable. The client may not necessarily share this information with the practitioner, perhaps because they may feel ashamed, afraid, or hesitant to disappoint the practitioner. Most often, the explicit focus of PPIs is not the amelioration of symptoms, but, rather, the promotion of wellbeing. Monitoring changing circumstances in the clients' lives is important, as is calibrating the PPIs to these changes. For example, if the client experiences a break-up or loses their job, it is important that the practitioner can address these changes and evaluate their impact. Practitioners should be prepared to suspend an ongoing PPI and adjust the approach accordingly to presenting and ongoing needs, or refer on to another helping professional if appropriate. Failure to do so can negatively impact the alliance, which may not be repairable.

\section{Changes in embedded environments.}

In addition to accounting for changes within clients, it is important to take into consideration the environment in which both clients and practitioners are embedded. Take a PPP who is also a clinician, as an example. Contemporary clinicians work in highly complex, fast-paced, and pressured environments which are marked by challenges such as a dearth of evidence-based treatment programmes, lack of cultural competence, and chronic and complex needs of clients that impact on multiple domains of their life. Given these challenges, consider clients who:

- may have lost their job or important contracts, or are being overlooked for a deserving promotion only because they do not fit the team;

- may have been recently diagnosed with a serious medical condition;

- are dealing with betrayal or other forms of mistreatment by their partner;

- are experiencing discrimination;

- are subject to destructive environmental events such as flooding or wildfire.

Consider how these challenges might affect clients and shape their treatments. These cases present different issues that need to be considered and addressed in different ways, all while considering the regulations and standards of the profession with which 
the person is aligned. Therefore, it is important that when PPPs offer PPIs, they learn about their clients, and also about their environments and recent or current changes to their circumstances. Practitioners also need to be cognisant of the challenges within their own work environment. This may include changes in administration which may bring changes in service modalities - for example, a new director may endorse or oppose certain PPIs or may prefer another treatment approach (e.g., mindfulness-based therapies or Acceptance Commitment Therapy). The changes may have direct or indirect impacts on the practice of PPIs, including from an ethical perspective.

\section{Current emotional state and level of wellbeing.}

While offering PPIs, it is important to continually assess clients' emotional state and level of wellbeing, and especially to monitor any changes for the worse. It is important that PPPs practice within their scope of expertise and training. For clients that present with issues related to psychopathology (i.e., depression, anxiety, trauma), then the PPP should refer on to a suitably qualified and experienced helping professional. If such issues are not present at the beginning of the working relationships, the PPP needs to be vigilant that such issues may surface while the client is engaged in a PPI and be ready to refer to a sufficiently qualified helping professional. Ideally, the practitioner would discuss with the client and consult with colleagues and relevant experts to ascertain what might be an appropriate timing for persisting with PPIs in such a case.

\section{Avoidance.}

Avoidance is one of the most common mechanisms people use to cope with negative emotions. A healthy psychological life entails being aware and adaptively using one's full emotional spectrum. Some clients seeking PPIs may avoid deeper negative emotions due to their discomfort, and may seek PPIs with hopes that they will dispel their negative emotions or keep them at bay. Examples of sources of negative emotions include memories of a bitter breakup or divorce, betrayal by a trusted friend, resentment for being the victim of discrimination, and being treated unfairly or in a biased manner by an individual or institution. One way to assess avoidance is by paying attention to negative emotions which find their way back in conversations despite not being discussed explicitly (i.e., emotions which persistently disturb clients and yet are deliberately not discussed).

Although PPIs are breaking new ground with increasing evidence that positive emotions and character strengths can ameliorate and repair negative states (Rashid \& Seligman, 2018; van Agteren et al., 2021), this is only the case if the negative states are meaningfully integrated in treatment. If strong negative emotions are avoided by clients or practitioners, the effectiveness of treatment may be compromised.

\section{Power differentials.}

A quintessential feature of any psychological interaction involving a practitioner and a client is the power dynamics inherent in the relationship, and especially the power differential. Therapists, counsellors, social workers, and other such professionals are usually aware of this differential. Therefore, they strive to create a safe, non-judgmental, confidential and welcoming space where clients do not feel like the mere passive recipients of prescribed services. Nonetheless, the nature of the transaction is such 
between the practitioner and the client that the practitioner is, inevitably, perceived as an expert. Clients tend to look up to them as equipped with state-of-the-art knowledge, training, and experience in delivering PPIs. Therefore, a power differential is always present. The practitioner must be cognisant of this differential and should avoid any situations where the client or practitioner may have to make a decision that might be influenced by this power differential.

\section{Overuse of strengths.}

Under or over use of some strength-based practices can exacerbate existing challenges. For example, in various coaching contexts, suggesting clients be more prudent can cause some to become more anxious or indecisive. Clients exercising forgiveness without deliberating around when and whom to forgive may overlook offences, which, if unchecked or unaccounted, can hurt others. Clients overusing creativity can find it challenging to adhere to norms or rules that foster equity and equal opportunity. Clients committed to making fair decisions in their workplace can find it hard to reconcile opposing realities existing in the same sphere (e.g., coming to terms with how a welleducated and well-informed person can make unwise decisions).

\section{Practical wisdom.}

Practitioners need what the philosopher Aristotle called "phronesis," i.e., practical wisdom to apply these guidelines. For example, consider a practitioner working with a high-level executive with whom they have established an excellent relationship and who has also made very good progress in their work. However, it comes to the practitioner's notice that the executive is involved in unethical behaviour. Should the practitioner preserve the solid relationship, or confront the person, risking the relationship? A single set of guidelines may not be sufficient. The practitioner needs to interpret the situation and balance competing motives. In such cases, practical wisdom is built from both cumulative experience and good relational bonds with colleagues who can advise and assist with ethical reasoning.

\section{Maintaining resilience and buffering against burnout.}

A number of PP constructs can buffer against practitioner burnout. Positive emotions (e.g., hope, serenity, joy, interest, and awe) can broaden a practitioner's attentional, cognitive, and behavioural resources, which can act as a buffer against vulnerabilities, such as symptoms of burnout (e.g., emotional exhaustion). Personal practices such as mindfulness can also help in that regard. Interpersonal strengths (e.g., social intelligence, kindness, love, humour, sense of purpose and meaning, and teamwork) can help cope with challenges such as depersonalisation (a state in which one's thoughts and feelings seem unreal or not to belong to oneself), as can a sense of purpose and meaning.

\section{Declaration of education and competence.}

Practitioners should declare the limits of their competence, and work within it, regarding specific psychological conditions, specific groups (e.g., working with children, other cultures, group work), and specific PPIs. For example, a practitioner may have experience in assessing strengths in adults, yet not with assessing children's or 
adolescents' strengths. Moreover, as the provision of service continues, it may move into areas in which the practitioner is less experienced or qualified. For example, whilst a practitioner may be experienced and qualified to provide services to all ages, they may not be experienced or qualified to deliver such services in the context of a family setting, in which case a referral or further support structures may be needed.

In addition, practitioners should declare the educational training and relevant experience upon which their provision of practice is based. For example, a practitioner completes a six-month certificate which included a one-week face-to-face interaction, with the remaining course completed online. The certificate is not recognised by a municipal, regional or provincial license granting authority. The practitioner's website states that they are a "certified positive psychology practitioner." They have no other professional practicing credentials. Is this ethical? It is important to explicitly inform your clients of the nature, scope and limits of your professional education, practice, and credentials beyond your working title.

Appreciating the importance of cultural context.

Ethical decision making should be sensitive to the circumstances of the cultural context. Such context is critical in the delivery of PPIs, because conceptualisation of what is normal, good, or adaptive is shaped by the values espoused as desirable by the culture and social context in which the concern or dilemma presents itself.

Additionally, cultures and social norms differ in how specific emotions are acknowledged, expressed, or amplified. Therefore, it is important that guidelines for an ethical practice of PPIs be rooted in such evidence. The ethical guidelines presented here are broad enough to accommodate cultural context, yet are sufficiently fine-grained to accommodate individual differences and needs. This integration of evidence, including cultural and individual factors, may be easy or readily available. Though such an integration may not yield an ethically perfect process or outcome, when individuals strive towards ethical excellence, the collective wisdom helps them to navigate cases as they are encountered. New ground is broken, new paths are paved, and eventually excellence can be achieved. Having the structure of these guidelines is, therefore, crucial to anchor a person's thinking and actions. These guidelines also serve the purpose of evaluating the extent to which one's actions are proximal or distant from ethical excellence. 


\section{Appendix B: Resolving Ethical Dilemmas - A Step-By-Step Guide}

Though it is impossible to be completely immune from being entangled in ethical dilemmas, the following steps, largely adapted from Ethics in Psychology (Koocher \& Keith-Spiegel, 1998), amongst other sources, will help a practitioner make sound ethical decisions:

- Practise in accordance with all statutes, regulations, and standards of professional practice, conduct, and guidelines, as stipulated by the respective license granting agency of your jurisdiction. Such information will help a practitioner navigate ethical dilemmas.

- Develop an accurate perception of how the same event may differ from person-toperson. Consult, with someone trusted and unbiased, as to whether the issue presents ethical dilemmas with serious consequences. Discuss with peers to see if what you are perceiving is an ethical infraction, merely poor professional etiquette, or a situation which poses harm to self or the public.

- Review already-published relevant guidelines, rules, and standards. Make sure you are interpreting these accurately and seek counsel if needed. Written guidelines are sometimes abstract and unclear, with ample room for subjective interpretation.

- Collect all relevant and the best information that you can. Identify existing gaps in information, and document facts and concerns.

- Integrate all the information in your process of reflection, and consult with someone who is well informed, open-minded, and unafraid to point out a practitioner's potential shortcomings.

- Evaluate how the six ethical principles of these guidelines apply (i.e., beneficence/nonmaleficence, responsible caring, respect for people's rights and dignity, trustworthiness, justice, autonomy). For example, evaluate the rights, responsibilities, and vulnerabilities of all involved.

- Brainstorm several solutions and appraise each as objectively as possible.

- Consider which character strengths might help you in making the soundest ethical decision. Might you turn to your deeper sense of fairness, honesty, or kindness? Perhaps your bravery is needed to motivate you to "do the right thing"? Remember that ethical decisions require the use of multiple character strengths used optimally and in combination, not just one strength in isolation or one strength "used a lot" (Niemiec, 2018).

- Visualise the outcome of your action or inaction.

- Reflect before making a decision on what impact it will have on you, all others involved, and what might be systematic implications. Also reflect on what kinds of support you may need to adhere to your decision.

- Translate the decision into concrete actions that truly reflect the spirit of your decision. A number of actions may express your decision. Select actions that express your decision accurately and validly, are measurable, connected to desired outcomes, and cause the least inconvenience or damage to those not directly involved.

Note: It is quite possible that the ethical principles may conflict with each other, or that a particular principle may take priority in a certain context. In such situations, consider strategies such as determining which values are more pertinent to the case; the long and short-term impact of prioritising one principle over another, or check in with peers on ways to resolve such conflicts. 


\section{Appendix C: Self-Evaluation Guide}

\section{Self-Evaluation Guide ${ }^{6}$}

The following section outlines an approach for practitioners to audit and reflect on their behaviours, attitudes, and practices to ensure ethical consideration, emphasising both client and practitioner wellbeing. These topics and questions could form part of regular self-reflective practise as discussion items to professional or within peer-supervision sessions.

Valuing ethics.

Practitioners are encouraged to view ethics as a helpful guide for promoting client wellbeing. Use the questions below to reflect on your attitudes and orientation toward ethical practice.

1. What is your perspective on the topic of "professional ethics?"

2. How well do you know the guidelines or code of ethics that informs your practice?

3. How often do you discuss ethical issues and decision making with trusted colleagues?

4. How frequently do you receive training in ethics?

5. What is your view on the ways that ethics informs responsible practice?

Example ethical dilemmas to reflect on.

\section{Mandatory reporting:}

How comfortable do you feel taking action against a client who might be responding well to PPIs, yet wishes for confidentiality when they inadvertently report an incident (recent or in the past) or ongoing situation which may involve, for example, child or elderly abuse, sexual abuse, or imminent threat to the safety of someone? If you are not practising under a code or guidelines of a professional body, do you have a legal requirement to report?

\section{Exaggeration and generalisation:}

You meet someone at a conference who appears passionate about PPIs and invites you to attend a workshop they are offering. You attend the workshop, which is built on a few studies with which you are quite familiar. The results, applications, and generalisability of studies are presented in an exaggerated manner. Most participants find the presentation "transformative" and appear to believe the findings. What would you do? Can you use the steps in the "resolving ethical dilemmas" section to guide your behaviour in such a situation?

\section{Application of PPIs.}

Practitioners are encouraged to be discerning in their application of positive psychological interventions. Use the questions below to reflect on your knowledge of and approach to using PPIs.

1. What are your criteria for deciding which PPI (e.g., gratitude journal, using specific strengths, forgiveness exercise) to use and with whom? Do you consider a client's presenting issues, circumstances, motivation for treatment, physical and mental abilities, personal attributes, preferences, and cultural and religious considerations?

2. How do you consider ways in which a PPI may not be an appropriate fit for the client?

\footnotetext{
${ }^{6}$ We are grateful to Dr Robert Biswas-Diener for his extensive knowledge, wisdom and contribution to the development of this self-evaluation guide.
} 
3. To what extent do you believe that PPIs might cause psychological harm? How? Under what circumstances?

4. What type of informed consent do you use before administering PPIs?

5. What steps do you undertake to decide when the PPI is appropriate? A) at the onset of the services, B) while the PPI has already commenced? In addition, under what conditions would you consider a concurrent or alternative service or treatment?

6. How regularly do you read primary source research about PPIs?

7. Using a specific example (e.g., gratitude interventions), how might you individualise PPIs to increase effectiveness and reduce potential harm?

\section{Reflective practice.}

Practitioners are encouraged to engage in reflective practice. This means that they reflect on their decision-making process, the process and outcomes of intervention, and seek feedback regarding their practice. Use the questions below to reflect on your ability to engage in this type of practice.

1. To what extent do you set aside time following a workshop or session with a client to reflect on your own process and performance?

2. What formal mechanisms do you have for capturing insights related to your practice and later implementing those insights?

3. How regularly do you solicit feedback from clients or colleagues regarding your practice?

4. Which colleagues do you most regularly seek out for discussion of ethics and best practices?

5. How frequently do you receive new training? How do you choose this training?

6. Are you aware of your own biases?

7. How do you know that you are being objective towards your clients.

\section{Self-care and burnout.}

Practitioners are encouraged to reflect on their health and wellbeing to protect against professional burnout and stress by engaging in ongoing self-care. Use the questions below to reflect on your self-care habits.

1. Being a practitioner does not make you immune from experiencing symptoms of mental illness that may move towards a clinical range. How aware are you of your mental health?

2. What steps or actions do you routinely engage in to take care of your mental health so that you can serve your clients optimally?

3. What are your predictable stressors, triggers, and professional vulnerabilities?

4. What barriers do you face in taking care of yourself? Do you consider things such as a lack of time and resources, feeling or believing "I am fine", and access to appropriate and relevant professionals who can take care of my mental health?

5. Who supports you? Who can you talk with about professional stress?

6. How would you know if you were experiencing burnout? What might you notice?

7. If you were to suffer emotional exhaustion or professional burnout, what would you be prepared to do to protect your clients and help yourself? 


\section{Appendix D: Case Examples of Ethical Issues}

The following cases are examples that may present in practice and have an ethical component relevant to the principles in these ethical guidelines. ${ }^{7}$

Case example 1: Cultural - applying PPIs with cultural sensitivity.

Ethical decisions should reflect the best available evidence in the circumstances of the cultural context. Consider the following vignette.

Suzanne is an Australian practitioner who is working with a female client of an East Asian cultural background. The client wants self-improvement and to preserve her family relationships. Suzanne opts to use the Best Possible Self Exercise. Suzanne follows all the steps to guide the client through the exercise. Somehow, despite making significant selfimprovements, the client reports that her relationships with her family have become sour. Hence, no significant improvement in the client's wellbeing is detected on repeated online measures.

An important implication in this vignette is consideration of the concept of the self, which varies from culture to culture. Suzanne follows a concept of self-development often associated with the West, which encompasses personal growth, and taking new and largely individual initiatives. By contrast, self-development in Eastern (and in most non-Western) cultures may place greater relative emphasis on investing in relationships, improving social interaction, and contributing to preserving family, group, and tribal traditions. Despite increasing cultural diversity in most urban metropolises, traditional cultural differences frequently still hold at least some sway. An important implication of these differences is that the client in this vignette may benefit from understanding how she can use her character strengths to bring benefit to her family or to connect with them more deeply. Suzanne may use the character strengths interventions of Turn Your Strengths Other-Oriented or Character Strengths Appreciation (Niemiec, 2018) in which her client learns to turn her best inner qualities into mechanisms of relationship building that benefits the other, and prioritises recognizing and valuing family members for their own best qualities. It could also be that self-development supported by significant others can bring deep and sustained increases in wellbeing. However, in an interdependent culture, self-development, which likely involves close family members, also requires managing more complex interactions over a longer period of time. By contrast, the Best Version of Me activity, which focuses on improving individual strengths or taking a new initiative, may require a relatively shorter amount of time. Therefore, it is imperative that PPPs consider how best their exercises can be culturally adapted.

The following ethical aspects are examples relevant to the above case:

- Responsible caring - The PPP needs to try to care for the client in a way that takes into account the specifics of that client's situation and background.

- Beneficence/non-maleficence - The PPP has a duty to do no harm.

- Respect for people's rights and dignity - The PPP has a responsibility to be sensitive to cross-cultural dynamics, and to respect the client's situated perspective and needs.

\footnotetext{
${ }_{7}$ We are grateful to the following individuals who provided these cases: Cases 1 and 3 - Tayyab Rashid, Case 2 Annalise Roache, Case 4 - Stewart Donaldson, Case 5 - Matthew Iasiello, Case 6 - Denise Quinlan, and Case 7 Robert Biswas-Diener.
} 
Case example 2: Coaching - coaching clients in changing circumstances.

Ethical dilemmas can appear unexpectedly and require an adjustment in service provision. Consider the following vignette.

Amanda came to coaching because she was feeling a lack of direction and meaning in life. Previously she had placed a lot of importance on career advancement, but now felt she needed to look for a more balanced approach to life, and moreover, was feeling sad and deflated that she had not accomplished more in life (outside of career enhancement). During the initial intake session, she identified several domains she would like to focus on, which included finance management (to support buying a home), personal development, and increasing fun and social time. She mentioned that her current job was challenging and that she would be looking to make a change in the future, yet this was not a current focus for coaching. In the first two sessions, in which goals were crafted and actions steps clarified, progress was being made in the direction she had indicated as important, and coaching was progressing well. However, at the beginning of the fourth session Amanda declared that her work situation had deteriorated abruptly and she had resigned, having found a new job already. The resignation had not been received well and Amanda was feeling unsettled and highly emotional in her workplace, where she still had four weeks to work out her notice. She indicates feeling triggered by her manager's reaction to her resignation, which is bringing up memories of similar emotional confrontations from her adolescences.

At this stage it was imperative for the coach to maintain their self-regulation strength and not make an impulsive suggestion due to the rise of Amanda's stressors. From there, the coach could then turn to using the strength of curiosity to check in on how Amanda would like to proceed in the immediate future, and be open to shifting focus within the session/s. It may also be important to understand the nature of Amanda's emotional reaction to determine if coaching is a safe and appropriate mechanism for her current needs. While the coach may have contracted Amanda for a set amount of sessions, the changes in the client's situation must inform the appropriate way forward.

The following ethical aspects are examples relevant to the above case:

- Responsible caring - The coach has a primary responsibility to use kindness and fairness to protect the welfare of Amanda and recognise the boundaries of their expertise.

- Autonomy - Here the coach could focus on developing Amanda's ability to be selfdirecting within the provision of services and all aspects of life, and to revisit/review the terms of their contract throughout the relationship.

- Beneficence/non-maleficence - The coach, acting in the best interests of Amanda and based on professional assessment, should be aware of working strictly within their limits of competence, and to provide services on the basis of adequate training and experience. 
Case example 3: Clinical - working with trauma.

Most individuals who experience trauma become overwhelmed in many culturally nuanced ways, especially if they are working on a PPI. They may develop extensive mechanisms to avoid thinking, recalling, or behaving in ways that may be related to their trauma. While offering PPIs, it is important to appraise and assess such trauma (whether historical and/or current). Without minimising or dismissing it, the practitioner ought to intervene if they have expertise, or, if not, to make a timely referral. The practitioner also needs to be vigilant in case trauma may surface while the client is engaged in a PPI. The practitioner must discuss with the client and consult with colleagues and relevant experts to ascertain what an appropriate timing might be for persisting with PPIs in such a case. Consider the following vignette.

Salma, 21, has a long history of experiencing physical, emotional, and sexual abuse. She seeks services for her persistent feelings of depression. She is expecting that the therapist would explicitly ask about the traumatic events in her life. Instead, she is given a short questionnaire which does not include any questions about current or past trauma. In the counselling sessions themselves, Salma finds she does not like the counsellor and chooses not to reveal the details of her trauma. Thus, the treatment remains superficially effective and she is prescribed a fresh round of treatment within six months.

As the above case demonstrates, it is important to explicitly ask about trauma (although doing so requires great sensitivity, and possibly also clinical training). Depending on the rapport, some clients will explicitly ask related questions, and seek advice, suggestions, coping strategies, or culturally appropriate resources. It is important that, while the PPI is in process and details of a recent or remote trauma surface, the practitioner responds in a way that meets the needs of the client. First and foremost, the practitioner should be sufficiently qualified, trained, and experienced in dealing with the situation. Practitioners should regularly tap their strengths of humility and prudence/caution by periodically checking if they should continue to provide services, or conversely, if the client would be better off with someone with more specific expertise in providing the suggestions, skills, and strategies that some clients need or ask for.

The following ethical aspects are examples relevant to the above case:

- Responsible caring - The PPP has a responsibility to care for Salma in a way that considers the specifics of her situation and background.

- Beneficence/non-maleficence - The PPP has both a responsibility not to create further harm to Salma, and to promote her wellbeing.

- Respect for people's rights and dignity - The PPP has a responsibility to be sensitive to cross-cultural dynamics, and to respect the client's situated perspective and needs.

Case example 4: Organisational - pressure to misrepresent data.

Many positive work and organisations interventions are evaluated to determine their effectiveness. Ethical dilemmas can appear unexpectedly when clients do not accept findings and pressure practitioners to omit or misrepresent evaluation data. Consider the following vignette and notice how a number of character strengths are underused, including honesty/integrity, perspective/wisdom, leadership, and humility. 
Company XYZ hired a PP practitioner to improve employee morale and performance. In consultation with company leadership, she decided to provide a training programme to increase employee psychological capital (hope, efficacy, resilience, and optimism).

Evaluation data showed the employees loved the training and wanted more positive psychological training in the future. However, the evaluation data showed there were not significant differences in levels of hope, efficacy, resilience, optimism, morale, or performance between employees who were randomly selected to participate in the training, and those in the comparison group. The leaders of Company $X Y Z$ were so pleased their employees enjoyed the training that they did not want them to see the discouraging evaluation results. Instead, they asked the practitioner to report that the training was a big success, and that more training along the same lines would be provided for the employees in the future.

As the above case demonstrates, practitioners may be asked to exaggerate the impact of positive psychology training to boost the employees' morale. However, in line with the principle of trustworthiness, practitioners should not omit or misrepresent evaluation data. To meditate the concerns around misrepresenting facts practitioners could focus on promoting the specific elements of the training that employees enjoyed. Furthermore, the practitioner should also carefully review and consult with someone with appropriate expertise in psychological capital to ensure the training adequately addresses the improvement of hope, efficacy, resilience, optimism, morale, and performance. The training content, outcome, and evaluation approach may need to be further refined to ensure they are appropriate and aligned with the desired outcomes.

The following ethical aspects are examples relevant to the above case:

- Autonomy - Here the practitioner's autonomy to act is being taken away.

- Beneficence/non-maleficence - Beneficence involves a commitment to promoting the client's wellbeing, and this level of deception advocated by the company's leaders is not in the best interests of the employees. Additionally, acting beneficently requires providing services based on adequate training or experience.

- Trustworthiness - Here the practitioner is being asked to exaggerate and generalise the effectiveness of the PPI, which does not honour the trust placed in the practitioner by the employees.

Case example 5: Mentoring - motivating "unwilling" participants in an imbalanced relationship.

Often, those who are most resistant to an intervention are those who may benefit most. PPPs encounter a difficult situation when trying to motivate or persuade a client to participate in an intervention for their benefit, without manipulation. Consider the following vignette.

Abbie, a case manager at a youth agency, has a client who refuses to participate in the exercises of a PP group intervention. The facilitator of the intervention has asked Abbie to motivate her client to participate in the exercises, and convince them of the benefits of the intervention. Abbie agrees with her colleague that the intervention would be greatly beneficial for her client. Abbie is conscious that her client's attitudes and behaviour often cause the client to be excluded from interventions that are likely to be helpful, and decides 
to challenge her client's attitudes towards participation. Abbie has developed a trusting, mentor relationship with the client, and is unwilling to compromise her integrity and betray the client's trust by blindly "selling" the intervention to the participant or making promises that won't be realised. Rather, she modestly describes the evidence that supports the intervention, and works with her client to weigh up the costs and benefits of participation. She curiously poses questions to her client about the pros and cons of participating in the intervention and how her client's signature strengths could be used in the group setting. Abbie then supports her client to identify strategies to minimise the costs of participation, and they practice low-intensity versions of the intervention itself and her client's strengths use prior to the group setting.

Abbie is certain that her client will benefit from participating in the intervention, and doesn't want her client to be excluded from participation once again. Equally, she does not want to betray her client's trust or autonomy. Abbie wants to challenge her client, and help expose her to new experiences, but fears that unethical manipulation could result in negative outcomes for her participant, and ultimately damage the quality of their hard-won relationship. Rather than over-selling or exaggerating the benefits of participation, Abbie works to understand the client's attitude toward the intervention, challenges cognitive biases, and creates opportunities to demonstrate the benefits of the training in the safety of the case management session.

The following ethical aspects are examples relevant to the above case:

- Justice - The PPP has a responsibility to ensure that clients are not excluded from interventions based on a particular characteristic.

- Trustworthiness - The PPP uses the trusting relationship with the client to encourage the client to evaluate their attitudes and beliefs, but does not betray this trust.

- Autonomy - The PPP respects the will of their client, and is hesitant to manipulate it.

Case example 6: Education - implementing wellbeing programmes where aspirations are hindered by existing practice.

Many schools want to adopt wellbeing programmes. However, they may do so for a range of reasons, from concern for teacher or student wellbeing, through addressing challenges like bullying within the school, to enhancing already strong practices. In addition, having a school wellbeing programme can also be viewed as a marketing opportunity and point of difference for some schools. Consider the following vignette.

A school staff member was requested by the principal to develop a wellbeing strategy and plan for adoption by the board, and then to organise a fast rollout of the key elements. The principal wanted to tell prospective parents that their children would benefit from explicit wellbeing teaching in the curriculum. Teachers were all assigned responsibilities for teaching wellbeing, even though there had been very little professional development for staff in this area. Many staff were stressed and felt unprepared for this teaching, as well as the additional workload it represented. A consultant was brought in to deliver a halfday professional development session in wellbeing for staff. Some staff expressed concern to the consultant that they were anxious that they might cause harm to their students because they were ill-equipped and had no grounding in this area. In addition, the consultant has seen the school's marketing which promotes its work on wellbeing (e.g., 
describing the school as supporting holistic wellbeing for staff and students, and building wellbeing at every encounter). The consultant is aware that the school continues to use a punitive discipline policy that undermines relationships between students and staff, and ignores scientific evidence for the wellbeing benefits of restorative practice and forgiveness. The mid-level teacher who has been made responsible for wellbeing programme implementation has briefed the consultant on these issues. The teacher is reluctant to raise the issues with the school leadership as this teacher has previously been intimidated by this group when raising similar concerns. At that time, their loyalty to the school was called into question, and their suitability to continue on as a teacher was challenged.

As the above case demonstrates, there can be many ethical issues layered in any complex situation. This case highlights issues related to autonomy, transparency, competency, accountability, safety, truthfulness, and trust, across four levels of the practitioner/consultant, school leadership, teacher and teaching staff, and students. With such complex cases and dynamics at play, it is important to survey the ethical principles that may underly these issues.

The following ethical aspects are examples relevant to the above case:

- Beneficence/non-maleficence - The PPP has a responsibility not to unequivocally or uncritically endorse a school as being a "wellbeing school" if they are aware of school practices that undermine wellbeing. They also have a duty of care if the teachers believe the program may cause harm if implemented inadequately.

- Autonomy - The teacher should not feel coerced into adopting a perspective or position that conflicts with their own judgement or values. The principle of autonomy opposes the manipulation of clients against their will, even for beneficial social ends, and it also emphasises the value of voluntary participation.

- Trustworthiness - the practitioner and teacher delivering the wellbeing program are having trust placed in them - by the client (the school) and by the students. As such they should, on the one hand, strive to ensure that expectations have reasonable prospects of being met, and on the other, are open and transparent about these prospects.

Case example 7 : Making statements about one's qualifications and about applied research.

Rapport and relationship quality between practitioner and client is essential in any service provision. This can be built with trust, truth and transparency. Consider the following vignette.

Ajit is a consultant who works with positive psychological approaches. His website states: "I am passionate about positive psychology, the science of human flourishing. Studies from this field prove that happiness is beneficial: it makes people healthier, more creative, and more sociable. As a result, I use a number of proven happiness interventions to help you boost your wellbeing (and your success!). In addition, I draw on the findings from neuroscience to help you hack your brain and be more successful."

Ajit is representing science in general and positive psychological science specifically. In doing so, he must take care to represent the results of research accurately, truthfully and transparently and to be cautious in his promises to potential clients. Specific considerations in his website that 
might need to be addressed include the language used, the claims being made, and the qualifications portrayed. For example, Ajit claims that research has proven that happiness is beneficial. "Proven" is a strong claim that is generally antithetical to the scientific method. More responsible alternatives include "research suggests" or "there is evidence for." As another example, while research suggests several benefits of happiness, the evidence is far less strong for creativity than health. The evidence is mixed that happiness causally leads to greater creativity. Ajit's language strongly suggests that the interventions he employs will lead to both higher wellbeing and success for his clients. Although they can influence these outcomes, he cannot responsibly promise them as such practitioners should be careful not to generalise or exaggerate research findings. Lastly, consumers of Ajit's services will assume, based on his statements, that he has some legitimate expertise in research and in positive psychology and neuroscience specifically. If it turns out that Ajit has simply read a few popular books on these topics, he does not indeed have the expertise and must be cautious in how he represents his qualifications.

The following ethical aspects are examples relevant to the above case:

- Trustworthiness - Ajit needs to be transparent and truthful about his qualification/s and experience in order to adequately set expectations that have a reasonable prospect of being met.

- Responsible caring: Ajit needs to be well aware of boundaries of his expertise and should make them very explicit as to what he is trained to do and what he is not, in terms of personal qualifications and specific experience in using positive psychology interventions within a specific domain (e.g., education, health, organizations, clinical) and with specific populations (e.g., children, adolescents, adults, couples, families, institutions).

- Autonomy - Ajit needs to be transparent and truthful about his qualification/s and experience in order to allow the client to have autonomy over their choices with regards to being self-directing in the provision of services. 


\section{Appendix E: Moving Towards a ‘Code of Ethical Positive Psychology Practice’?}

As mentioned in the preamble above and as indicated in Figure 1 there, 'guidelines' usually sit within broader frameworks of ethical practice, which will vary by context and jurisdiction.

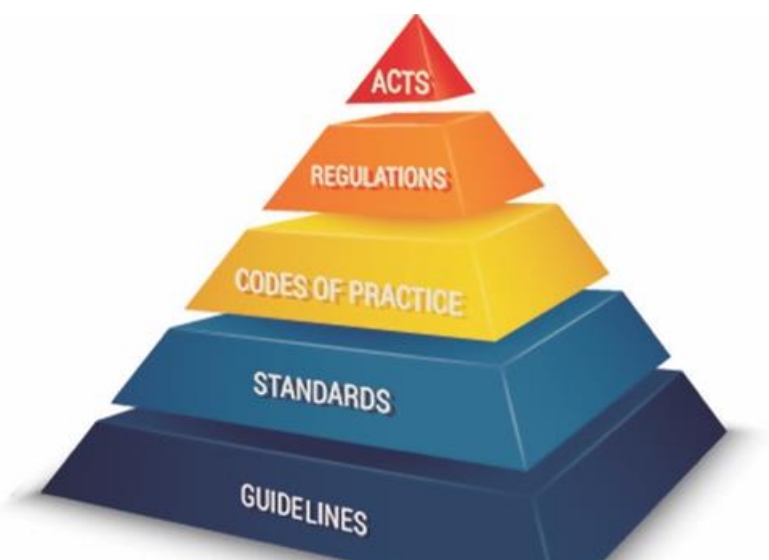

Being at the bottom of a prescriptive hierarchy, it is important to consider guidelines along with other relevant frameworks. Although definitions will vary, 'guidelines' usually refer to guidance developed by subject matter experts, and will use more suggestive language such as "may" or "it is suggested or recommended that". Above guidelines are 'standards' which are typically completed in a consensus process and approved by a certification body, with the language of standards being both suggestive (e.g., "suggested that") and mandatory (e.g., "will"). At one level higher 'codes' indicate what one must do, are adopted by a regulatory body, and include language such as "shall" and "will" which indicate that action is mandatory. As such, codes have enforceable ramifications that are regulated and policed. As also mentioned in the introductory notes, codes supersede guidelines, and where a practitioner is a member of a relevant professional body, then first and foremost their association's standards or code (or guidelines) will inform their practice.

Therefore, the next step in moving from guidelines towards a 'code' of practice would be the adoption of these 'ethical guidelines for positive psychology practice' by a regulatory body that can enforce them. This may also involve a credentialing and registration process for members associated with that body. When such a point arrives, the code itself will likely require aspects such as mandatory reporting, and adoption of more forceful, stringent language, and greater clarification of behaviour examples. However it is also noteworthy that these guidelines were developed in the most part from various codes, rather than guidelines, as indicated in Appendix G. 


\section{Appendix F: Strengths in Ethics}

Positive Psychology is the scientific endeavour about what makes life worth living and what goes right in life. It explores qualities, traits or attributes which create effective, inclusive, accessible and ethical practices at work, in education, health and leisure. There are several frameworks of these qualities, traits and attributes. It is our view that utilising strengths can promote ethical practice.

The intention of this section is to aid in moving the guidelines to becoming more strength neutral (i.e., relevant across many frameworks). The guidelines themselves are infused with strengths from the VIA Classification of Character Strengths and Virtues framework. This framework (Peterson \& Seligman, 2004) comprises 24 intrinsically fulfilling, ubiquitous traits, valued across cultures, and viewed as central components of a fulfilling life. The framework is reported to be the most used strengths framework by PPP's, cross-culturally validated, available in many languages, and accessible (free basic assessment). The VIA website (www.viacharacter.org) lists more than 300 studies which have used this framework.

\section{Considerations for using strengths to support ethical decision making.}

Whilst iteration two of the Ethical Guidelines utilises the VIA framework (Peterson \& Seligman, 2004), one could equally apply others strengths frameworks to support ethical practice. Such frameworks might be the CliftonStrengths (Clifton \& Anderson, 2002) or the Strengths Profile (Linley, 2008) as examples. Whereas the VIA ask the question "who are you?" and investigates character, the CliftonStrengths asks "what do you do well?" and identifies talents, and the Strengths Profile asks "what energizes you?". The use of other frameworks can be enacted by adapting the terminology a specific strengths based framework with the strengths highlighted in the guidelines. The key point is to harness strengths (regardless of specific terminology) to support ethical practice and decision making.

\section{Links to three models.}

- VIA Classification of Virtues and Strengths https://www.viacharacter.org/

- The CliftonStrengths https:/www.gallup.com/cliftonstrengths/

- The Strengths Profile https://www.strengthsprofile.com/ 


\section{Appendix G: Relevant Guidelines}

The following guidelines (organised by specific field and alphabetised within the field) were drawn upon and helped inform the development of these practice guidelines:

Psychological:

- Australia - Psychology - Australian Psychological Society (APS), Code of Ethics (2017).

- Australia - Counselling and Psychotherapy - Psychotherapy \& Counselling Federation of Australia (PACFA), Interim Code of Ethics (2015).

- Canada - Psychology - Canadian Psychology Association (CPA), Canadian Code of Ethics for Psychologists (2017).

- New Zealand - Psychology - New Zealand Psychological Society (NZPS), Code of Ethics for Psychologists (2012, 4th Ed).

- United Kingdom - Psychology - The British Psychological Society (BPS), Practice guidelines (2017, $3^{\text {rd }}$ Ed).

- United Kingdom - Psychology - The British Psychological Society (BPS), Code of Conduct (2018).

- United Kingdom - Counselling and Psychotherapy - British Association for Counselling and Psychotherapy (BACP), Ethical Framework for Good Practice in Counselling and Psychotherapy (2010).

- United States of America - Psychology - American Psychological Association (APA), Ethical Principles of Psychologists and Code of Conduct (2017).

- United States of America - Counselling - American Counselling Association (ACA), Code of Ethics (2014).

Nursing:

- Australia - Nursing - Nursing and Midwifery Board of Australia: Code of Ethics for Nurses (2008).

- New Zealand - Nursing - Nursing Council of New Zealand (NCNZ), Code of Conduct for Nursing (2012).

United Kingdom - Nursing - Nursing and Midwifery Council (NMC), The Code for Nurses and Midwives (2015).

Coaching:

- International - Coaching - The International Coaching Federation (ICF): Core Competencies and Code of Ethics (2018). 